\title{
Determination of the Optimum Moisture Content of Purple Ipê (Handroanthus heptaphyllus) Seeds for Biospeckle Tests
}

\author{
Juliana Aparecida Fracarolli ${ }^{1}$, Adilson Machado Enes ${ }^{2}$, Inácio Maria Dal Fabbro ${ }^{3}$ and Leila Martins ${ }^{4}$ \\ 1. Department of Postharvest Technology, School of Agricultural Engineering, University of Campinas, Campinas, Sao Paulo \\ 13083-875, Brazil \\ 2. School of Agricultural Engineering, Federal University of Sergipe, São Cristóvão 49100-000, Sergipe, Brazil \\ 3. Department of Farm Machinery, School of Agricultural Engineering, University of Campinas, Campinas 13083-875, Sao Paulo, \\ Brazil \\ 4. Technical Assistance Coordination Integral (CATI), Campinas 13070-178, Sao Paulo, Brazil
}

\begin{abstract}
Handroanthus heptaphyllus (botany synonymy Tabebuia heptaphylla) is considered to have very important economical, ornamental and medicinal value. It is included in the Bignoniaceae botanical family, whose multiplication is quite solely carried by seeds. Forest dispersion elements, i.e., the seeds, deserve a deep understanding on their physiological performance. The objective of this research work was to determine the optimum seed moisture content for laser interaction in biospeckle tests, which is based on laser interferometry. Seeds were hydrated to the level of $69 \%$ and then divided into four subsamples, i.e., (1) seeds without teguments, (2) seeds with tegument, (3) seeds without teguments frozen at $0{ }^{\circ} \mathrm{C}$ and (4) seeds with teguments frozen at $0{ }^{\circ} \mathrm{C}$. The four subsamples were then separated into five moisture level groups $(28 \%, 35 \%, 54 \%, 63 \%$ and $69 \%)$ and exposed to the laser. The biospeckle phenomenon quantification was carried out by means of the moment of inertia (MI) approach. A positive correlation between MI and seed moisture content was obtained. It was also verified that seed moisture content between $28 \%$ and $54 \%$ showed better interaction with the laser. The results are important for the application of the biospeckle technique as a possible tool to assess the quality of purple ipê seeds.
\end{abstract}

Key words: Purple ipê (Handroanthus heptaphyllus), seed moisture content, biospeckle, moment of inertia.

\section{Introduction}

New methods of seed analysis have been discussed as novel proposals for research topics [1,2]. Nuclear magnetic resonance, near-infrared hyperspectral imaging, X-ray analysis and response to light stimuli are included among these scientific reports to assess the seed quality and viability [3-7]. Optical methods applied to the analysis of biological materials, which are based on artificial vision or machine vision and image processing techniques, should be mentioned as well. A new technique called biospeckle or dynamic speckle has also been highlighted among the optical

Corresponding author: Juliana Aparecida Fracarolli, professor, research field: postharvest technology. methods, which is related to an optical interference phenomenon capable of carrying information about material exposed to a coherent light source. Based on research on biological materials, specifically on seeds, there are now well-defined methodologies for measuring and quantifying the above referred phenomenon in laboratory condition. The main features of the proposed test are associated to its non-destructive character, non-subjectivity and automation capability. Although there are many optical techniques that can be used with automation capability, the proposed technique in this research work is biospeckle, whose physical principle is the interference phenomenon that occurs when a coherent 
beam of light reflects from a rough surface. The biospeckle activity corresponds to microscopic activity of biological samples. Braga et al. [8] has explained that processes related to movement of the scattering centers in the tissue is due to organelle vibration, cytoplasmic streaming, cell growth and multiplication, and biochemical reactions that are responsible for certain biospeckle activity.

Information about the activity of a biological material through biospeckle can be obtained via different methods. There are methods that provide visual results, such as Fujii and generalized differences (DG). There are also methods that generate numerical results, such as moment of inertia (MI), contrast method and auto correlation. The calculation of MI is based on the temporal variation of the speckle. To quantify the temporal variation of the speckle, spatial temporal speckle (STS) is used. There are research works that present new methods for biospeckle analysis, such as modifications in MI [9-12], frequency responses and absolute value of the differences (AVD) [13], normalization of co-occurrence matrix [14]. Recently, cross-correlation coefficient has been used for the analysis of fruit quality $[15,16]$.

The purple ipê (Handroanthus heptaphyllus) is an arboreal species, native of South America. Among its main features, the wood properties, ornamental qualities and leaf extracts should be included. In the case of endangered native species, it becomes necessary to undertake studies directed to preserve the species, facilitating their use and spread on rehabilitation and reforestation areas. Thus, considering that the main spreading way of purple ipê is carried by seeds and that forest seeds analysis methodologies are not well consolidated and normalized, the search for adequate methods of seed analysis assumes vital role for the success of forestry programs involving those species. Seed water absorption kinetics, in general, consists of three phases. The first one is identified as soaking, which is generated from the matric potential of cell contents and cell walls. It occurs in dormant, non-dormant, viable and non-viable seeds. The second phase also occurs with all seeds. However, the third phase, which is associated with root protrusion, occurs only with viable seeds [17]. Seed water content is related to the intensity of metabolic processes, which, in turn, affects the temporal variation at the points of laser incidence in biospeckle test, as noted by Rodrigues et al. [18]. It is equivalent to state that the biological material moisture is related to MI values. Rodrigues et al. [18] used biospeckle techniques to differentiate living tissues of bean seeds from dead tissues by means of freezing water. The results showed that for the same water content, it is possible to differentiate living seeds from seeds having reduced potential due to the formation of water crystals. According to Nobre et al. [11], the MI value characterizes the activity by assigning a number to its state. The authors evaluated the spectral components of the STS by means of the MI, as well as the entropy expressed through wavelet and cross-spectrum analysis. Based on what was exposed, the objective of this study was to determine the optimum moisture content of purple ipê seeds for biospeckle tests.

\section{Material and Methods}

\subsection{Places, Sample Acquisition and Equipment Employed}

Biospeckle, seed viability and vigor tests were carried out at the Optics and Postharvest Laboratories of the School of Agricultural Engineering, University of Campinas (FEAGRI/UNICAMP), Campinas, Sao Paulo, Brazil. Purple ipê (Handroanthus heptaphyllus) seeds were obtained from 15 donor trees at the Seedlings Production Center, Department of Seeds, Seedlings and Matrices (DSSM), Integral Technical Assistance Coordination (CATI) in Pederneiras, Sao Paulo, Brazil. The fruits, collected mature and closed, were placed under shade in order to remove the excess moisture for subsequent seed extraction. The 
experimental setup for optical tests included: a 632 $\mathrm{nm}$ wavelength (red) and $10 \mathrm{~mW}$ diode laser as a light source, a digital camera with an image acquisition rate of $60 \mathrm{~Hz}$, a personal computer with a Quad Core processor and $4 \mathrm{~GB}$ of random access memory (RAM) with software for image processing, namely SUPER(C) version 2012.bld.54 in order to break the videos into frames and ImageJ version 1.47q [19] to process the images and to obtain the MI, statistical software Sisvar version 5.3 [20] and R version 3.0.0 [21].

\subsection{Sample Preparation}

In order to characterize the adequate moisture content range for optimum interaction between the laser and seeds, a sample of 100 seeds were immersed in water with plastic bags for $24 \mathrm{~h}$ to increase the seeds' moisture. Then, four subsamples were obtained and forwarded to four treatments, as follows: T1 -25 seeds with teguments removed, T2-25 seeds with tegument preserved, T3-25 seeds with tegument removed and subjected to a low temperature with a freezer $\left(0{ }^{\circ} \mathrm{C}\right)$ for $12 \mathrm{~h}$ and $\mathrm{T} 4-25$ seeds with tegument and also subjected to $0{ }^{\circ} \mathrm{C}$ for $12 \mathrm{~h}$. The initial moisture content of subsamples was measured in an oven at $130 \pm 3{ }^{\circ} \mathrm{C}$ for $2 \mathrm{~h} \mathrm{[22],} \mathrm{and} \mathrm{from} \mathrm{that}$ value, the other moisture content values were obtained by weighing the samples on precision scales at intervals of $3 \mathrm{~h}$.

\subsection{Optical Tests}

To expose the seeds to the laser for the optical test, they were fixed in an acrylic container. The container was weighed before and after the seeds were fixed in order to determine the seeds' moisture content. The seeds from treatments $1,2,3$ and 4 have undergone the same experimental procedures in optical testing. After the lighting, the images were processed to obtain the MI values for each seed at five different moisture levels $(28 \%, 35 \%, 54 \%, 63 \%$ and $69 \%)$.

The seeds were illuminated by the laser in random order, which was obtained through a previous draw.
During the lighting, $10 \mathrm{~s}$ films were made for each seed in each treatment.

Fig. 1 illustrates the experimental configuration adopted in the tests with biospeckle laser. The angle of incidence of light was about 45 degrees.

For the biospeckle test, 512 successive video frames of $640 \times 480$ pixels were captured for each seed and forwarded for image processing, including the color conversion to 8 bits with 256 levels of gray. Then, MI values were generated through the ImageJ software.

\subsection{The Experimental Design}

A randomized design was adopted with 25 replications in a $4 \times 5$ factorial with the four treatments and five moisture levels. The results were subjected to analysis of variance by $F$ test and the averages were compared by Tukey test at 5\% probability, using the program Sisvar 5.3.

The adopted response variables were identified as MI values. Table 1 shows the details of the statistical factors as well as the factor levels used in this experiment.

\section{Results and Discussion}

Analysis of variance of the MI values obtained from the seeds of Handroanthus heptaphyllus showed a significant difference between seeds with teguments and seeds without tegument, and seeds subjected to freezing and seeds not subject to freezing for the variable MI. The MI values showed to be efficient in differentiating the four treatments, except for the humidity level of $69 \%$, in which $\mathrm{T} 3$ and $\mathrm{T} 4$ had statistically identical averages, as shown on Fig. 2.

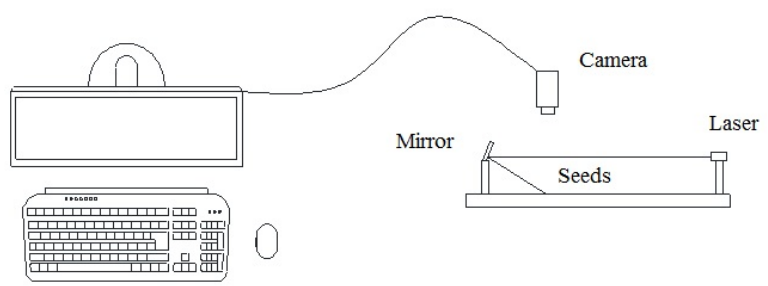

Fig. 1 Adopted experimental setup for the biospeckle tests. 
Table 1 Factors, levels and treatment adopted for the experiment.

\begin{tabular}{llll}
\hline Factors & Amount of levels & No. & Treatments \\
\hline & & 1 & Seeds without tegument \\
Damage to plant tissue and & 4 & Seeds with tegument \\
tegument influence & 3 & Seeds without tegument and submitted to freezing \\
& & 4 & Seeds with tegument and submitted to freezing \\
\hline & & $28 \%$ \\
Seed moisture content & 5 & $35 \%$ \\
& 3 & $54 \%$ \\
& 4 & $63 \%$ \\
& 5 & $69 \%$ \\
\hline
\end{tabular}

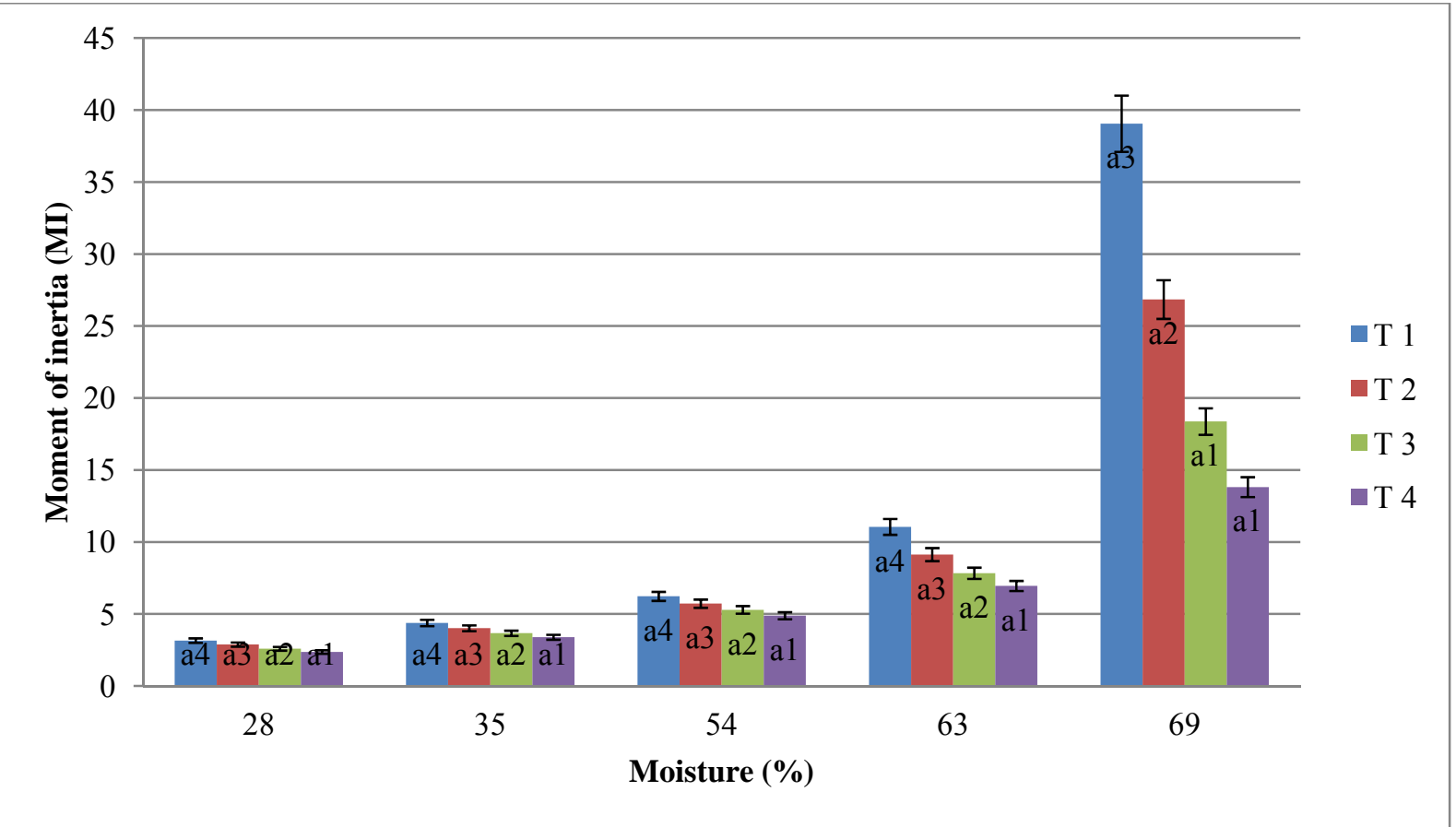

Fig. 2 Average MI values for each studied moisture treatment.

T1: seeds without tegument; T2: seed with tegument; T3: seeds without tegument and subjected to freezing at $0{ }^{\circ} \mathrm{C}$; T4: seed with tegument and subjected to freezing at $0{ }^{\circ} \mathrm{C}$.

Averages followed by the same letter and number within each moisture do not significantly differ by Tukey test at $5 \%$ probability.

For all the moisture content $(28 \%, 35 \%, 54 \%, 63 \%$, and $69 \%$ ), T1 presented the highest MI values. It is due to the fact that in those samples, the seed endosperm was observed with biospeckle. The endosperm is a reserve tissue, and when it is hydrated, cell multiplication, cellular respiration, mitochondria and chloroplasts activation, and protein synthesis occur. A decrease of starch content also occurs in order to provide energy for the seedling development. All of those reactions represent movements in the cytoplasm, which are recorded by biospeckle and provide a higher quantification of MI than the one recorded by lighting the seeds with tegument. On the other hand, in T2 where the seeds were observed with tegument, those samples showed lower MI values than $T 1$, because the tegument is not a living tissue. In the teguments, organelle movement or cell multiplication do not occur. The tegument cells are basically formed with lignin, which is an important structural material constituted by organic polymer. It also influences the biospeckle response. T3 and T4 showed lower MI values due to the fact that the seeds 
were not living. When the seeds were submitted to freezing, ice crystals have broken the cell walls, killing the cells and seeds. So the movement due to biochemical reactions in living tissue did not occur. It could be noticed in biospeckle activity with lower MI values.

The average MI values, as well as the statistical details, are shown in Table 2. The significance level $(\alpha)$ and coefficient of variation (CV) indicate that the most appropriate seed moisture range falls between $28 \%$ and $54 \%$ for the biospeckle test to be carried out. Higher moisture content may have influenced the MI values, resulting in higher CV. It is due to the fact of water evaporation occurring in samples with high moisture content. A gradient of vapor pressure between the environment relative humidity and the seed sample occurs, leading to water evaporation.

The obtained information is of fundamental importance for improving the efficiency of the application of the biospeckle technique on ipê seeds, since they indicate the optimum moisture content to carry out the tests. MI values were found to be a function of the moisture content, as reported by Rodrigues et al. [18]. The higher is the moisture, the higher is the MI values. In Fig. 3, the average MI values are represented on the ordinate, and the moisture levels are represented on the abscissa for each assessed treatment.

The correlation between the MI and the moisture content values was carried by means of the linear model function in software R. It was possible to adjust a linear model between moisture and MI values for each of the treatments until the moisture content of $63 \%$. After that, it was done through the analysis of variance and Pearson's test to assess the significance of the correlation.

The correlation between moisture content and MI values for each treatment, level of significance, $R^{2}$ value, $P$ value, as well as the regression line equation are displayed on Table 3.

Table 2 Statistical results (ANOVA and Tukey test) for the average MI values of seeds from each treatment.

\begin{tabular}{|c|c|c|c|c|c|c|c|}
\hline Moisture & Treatments & Averages MI & MSD & CV (\%) & $F$ & Significance level & No. of observations \\
\hline \multirow{4}{*}{$28 \%$} & $\mathrm{~T} 1$ & $3.15(\mathrm{a} 4)$ & \multirow{4}{*}{0.105} & \multirow{4}{*}{5.12} & \multirow{4}{*}{149.411} & \multirow{4}{*}{0.00001} & \multirow{4}{*}{100} \\
\hline & $\mathrm{T} 2$ & 2.88 (a3) & & & & & \\
\hline & $\mathrm{T} 3$ & $2.59(\mathrm{a} 2)$ & & & & & \\
\hline & $\mathrm{T} 4$ & $2.36(\mathrm{a} 1)$ & & & & & \\
\hline \multirow{4}{*}{$35 \%$} & $\mathrm{~T} 1$ & $4.38(\mathrm{a} 4)$ & \multirow{4}{*}{0.147} & \multirow{4}{*}{5.12} & \multirow{4}{*}{117.869} & \multirow{4}{*}{0.00001} & \multirow{4}{*}{100} \\
\hline & $\mathrm{T} 2$ & $4.01(\mathrm{a} 3)$ & & & & & \\
\hline & $\mathrm{T} 3$ & $3.66(\mathrm{a} 2)$ & & & & & \\
\hline & $\mathrm{T} 4$ & $3.39(\mathrm{a} 1)$ & & & & & \\
\hline \multirow{4}{*}{$54 \%$} & T1 & $6.23(\mathrm{a} 4)$ & \multirow{4}{*}{0.220} & \multirow{4}{*}{5.35} & \multirow{4}{*}{95.284} & \multirow{4}{*}{0.00001} & \multirow{4}{*}{100} \\
\hline & $\mathrm{T} 2$ & $5.72(a 3)$ & & & & & \\
\hline & $\mathrm{T} 3$ & $5.29(\mathrm{a} 2)$ & & & & & \\
\hline & $\mathrm{T} 4$ & $4.88(\mathrm{a} 1)$ & & & & & \\
\hline \multirow{4}{*}{$63 \%$} & $\mathrm{~T} 1$ & $11.05(\mathrm{a} 4)$ & \multirow{4}{*}{0.629} & \multirow{4}{*}{9.67} & \multirow{4}{*}{111.291} & \multirow{4}{*}{0.00001} & \multirow{4}{*}{100} \\
\hline & $\mathrm{T} 2$ & $9.13(\mathrm{a} 3)$ & & & & & \\
\hline & $\mathrm{T} 3$ & $7.83(\mathrm{a} 2)$ & & & & & \\
\hline & $\mathrm{T} 4$ & $6.95(\mathrm{a} 1)$ & & & & & \\
\hline \multirow{4}{*}{$69 \%$} & $\mathrm{~T} 1$ & $39.06(\mathrm{a} 3)$ & \multirow{4}{*}{4.718} & \multirow{4}{*}{25.86} & \multirow{4}{*}{76.508} & \multirow{4}{*}{0.00001} & \multirow{4}{*}{100} \\
\hline & $\mathrm{T} 2$ & $26.84(\mathrm{a} 2)$ & & & & & \\
\hline & $\mathrm{T} 3$ & 18.37 (a1) & & & & & \\
\hline & $\mathrm{T} 4$ & $13.82(\mathrm{a} 1)$ & & & & & \\
\hline
\end{tabular}

T1: seeds without tegument; T2: seed with tegument; T3: seeds without tegument and subjected to freezing at $0{ }^{\circ} \mathrm{C}$; $\mathrm{T} 4$ : seed with tegument and subjected to freezing at $0{ }^{\circ} \mathrm{C}$; MSD: minimum significant difference; $\mathrm{CV}$ : coefficient of variation.

Averages followed by the same letter and number within each seed moisture content do not differ by Tukey test at $5 \%$ probability. 


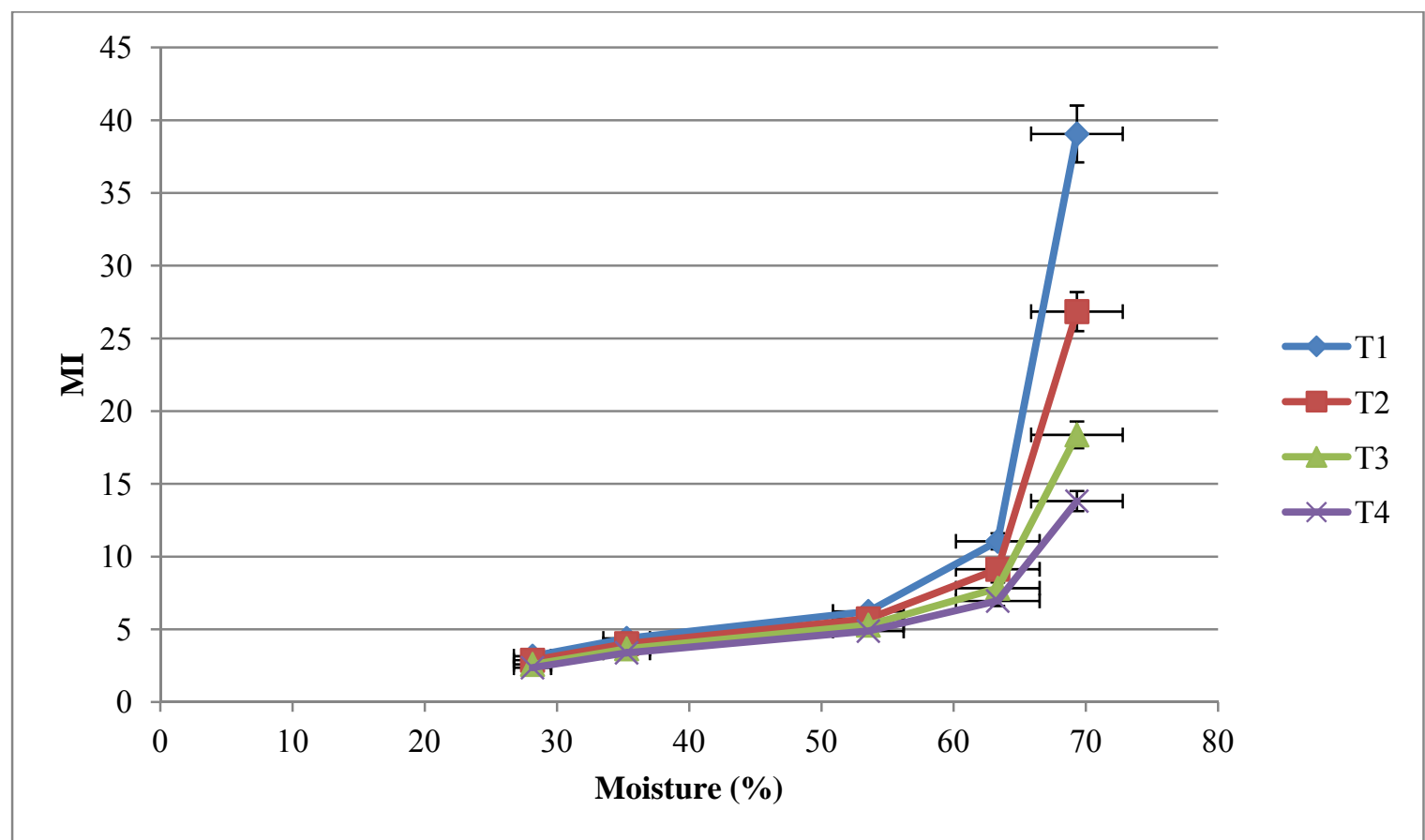

Fig. 3 Average MI values for each moisture content for each treatment.

T1: seeds without tegument; T2: seed with tegument; T3: seeds without tegument and subjected to freezing at temperatures of $0{ }^{\circ} \mathrm{C}$; T4: seeds with tegument and subjected to freezing at temperatures of $0{ }^{\circ} \mathrm{C}$.

Table 3 Results of the correlation between seed moisture content and average MI values.

\begin{tabular}{llllll}
\hline Treatment & Significance level (\%) & Correlation coefficient & $R^{2}$ & $P$ value & Regression equation \\
\hline 1 & 10 & 0.9305 & 0.8658 & 0.06950 & $y=4.362 x+17.947$ \\
2 & 5 & 0.9528 & 0.9078 & 0.04723 & $y=5.686 x+14.096$ \\
3 & 5 & 0.9698 & 0.9404 & 0.03024 & $y=6.919 x+11.494$ \\
4 & 5 & 0.9767 & 0.9540 & 0.02327 & $y=7.973 x+9.957$ \\
\hline
\end{tabular}

In the regression equations, $y$ stands for moisture content, while $x$ stands for MI values. The higher $R^{2}$ value associated to T4 indicates good linear adjustment between MI and seed moisture content. It can be noted through the value of $R^{2}$ (coefficient of determination) that for $\mathrm{T} 4$ where the seeds were with tegument subjected to freezing at temperatures of $0{ }^{\circ} \mathrm{C}$, the correlation between MI values and seed moisture content obtained experimentally was closer to the linear adjustment proposed by the mathematical model.

The correlation coefficient between the observed responses and those predicted by the model was also higher for T4 (0.9767). The lowest value was obtained for T1 (0.9305), in which the seeds without tegument.

A positive linear correlation between MI values and the values of ipê seeds' moisture content was obtained, as shown on Table 3. The water content is directly related to MI values, which are in close agreement with the results reported by Rodrigues et al. [23]. However, the linear and positive correlation was observed on values of moisture content up to $63 \%$. From this value, seed moisture content exhibited nonlinear behavior.

The CV associated to agricultural experiments are classified as low if they are under $10 \%$, medium when they fall between $10 \%$ and $20 \%$, high when they are included between $20 \%$ and $30 \%$, and very high when they are above $30 \%$. Research work performed on biospeckle tests has shown that $\mathrm{CV}$ was relatively high when compared with traditional agricultural experiments, as can be seen in the report by Braga et 
al. [1], where the obtained CV was $21.99 \%$.

Observing the statistical results presented on Table 2, it can be concluded that, in this experimental work, higher CV (25.86\%) were only found for seed moisture content of $69 \%$.

\section{Conclusions}

From this study, it can be concluded that the most suitable moisture content for laser and purple ipê seed interaction falls between $28 \%$ and $54 \%$. There was a positive correlation between moisture content and MI values for seed moisture content of up to $63 \%$.

This information is important for future research with biological tissue assessment with the biospeckle technique and its application on ipê seeds, since they indicate the optimum moisture content for the tests to be carried out.

Similar tests can be done with different species in order to determine the optimum moisture content for performing the biospeckle tests. Also, other methods of obtaining information through biospeckle should be tested, in order to obtain lower $\mathrm{CV}$, like absolute value of differences (AVD), the modifications in MI and cross-correlation coefficient. In future work, an automatic test can be developed to assess seeds based on biospeckle, which consists of a low cost, non-invasive and non-destructive technique.

\section{Acknowledgments}

The authors acknowledge the School of Agricultural Engineering of the University of Campinas, UNICAMP, the Laboratory of Farm Machinery, as well as the Laboratory of Postharvest Technology, the Laboratory of Seeds, Seedlings and Donor Trees, LSMM/CATI, and the National Research Council, CNPq, for the financial support.

\section{References}

[1] Braga, R. A., Dal Fabbro, I. M., Borem, F. M., Rabelo, G., Arizaga, R., Rabal, H. J., and Trivi, M. 2003. "Assessment of Seed Viability by Laser Speckle Techniques.” Biosyst. Eng. 86 (3): 287-94.
[2] Rabelo, G. F., Enes, A. M., Braga, R. A., and Dal Fabbro, I. M. 2011. "Frequency Response of Biospeckle Laser Images of Bean Seeds Contaminated by Fungi." Biosyst. Eng. 110 (3): 297-301.

[3] Foucat, L., Chavagnat, A., and Renou, J. P. 1993. "Nuclear Magnetic Resonance Micro-imaging and X-Radiography as Possible Techniques to Study Seed Germination." Sci. Hortic. 55 (3-4): 323-31.

[4] Kobori, N. N., Cicero, S. M., and Medina, P. F. 2012. "X-Ray Test to Evaluate the Quality of Castor Seeds." Journal of Seed Science. 34: 125-33. (in Portuguese)

[5] Krishnan, P., Joshi, D. K., Nagarajan, S., and Moharir, A. V. 2004. "Characterisation of Germinating and Non-germinating Wheat Seeds by Nuclear Magnetic Resonance (NMR) Spectroscopy.” Eur. Biophys. J. 33 (1): 76-82.

[6] Pinto, T. L. F., Cicero, S. M., França-Neto, J. B., and Forti, V. A. 2009. "An Assessment of Mechanical and Stink Bug Damage in Soybean Seed Using X-Ray Analysis Test." Seed Sci. Technol. 37 (1): 110-20.

[7] Shrestha, S., Knapič, M., Žibrat, U., Deleuran, L. C., and Gislum, R. 2016. "Single Seed Near-Infrared Hyperspectral Imaging in Determining Tomato (Solanum lycopersicum L.) Seed Quality in Association with Multivariate Data Analysis." Sens. Actuators B Chem. 237: 1027-34.

[8] Braga, R. A., Dupuy, L., Pasqual, M., and Cardoso, R. R. 2009. "Live Biospeckle Laser Imaging of Root Tissues." Eur. Biophys. J. 38 (5): 679-86.

[9] Sendra, G. H., Arizaga, R., Rabal, H., and Trivi, M. 2005. "Decomposition of Biospeckle Images in Temporary Spectral Bands." Opt. Lett. 30 (13): 1641-3.

[10] Skipetrov, S. E., Peuser, J., Cerbino, R., Zakharov, P., Weber, B., and Scheffold, F. 2010. "Noise in Laser Speckle Correlation and Imaging Techniques." Opt. Express 18 (14): 14519-34.

[11] Nobre, C. M. B., Braga, R. A., Costa, A. G., Cardoso, R. R., Da Silva, W. S., and Sáfadi, T. 2009. "Biospeckle Laser Spectral Analysis under Inertia Moment, Entropy and Cross-Spectrum Methods." Opt. Commun. 282 (11): 2236-42.

[12] Cardoso, R. R., Costa, A. G., Nobre, C. M. B., and Braga, R. A. 2011. "Frequency Signature of Water Activity by Biospeckle Laser." Opt. Commun. 284 (8): 2131-6.

[13] Braga, R. A., Nobre, C. M. B., Costa, A. G., Sáfadi, T., and Da Costa, F. M. 2011. "Evaluation of Activity through Dynamic Laser Speckle using the Absolute Value of the Differences." Opt. Commun. 284 (2): 646-50.

[14] Cardoso, R. R., and Braga, R. A. 2014. "Enhancement of the Robustness on Dynamic Speckle Laser Numerical Analysis." Opt. Lasers Eng. 63: 19-24. 
[15] Kurenda, A., Pieczywek, P. M., Adamiak, A., and Zdunek, A. 2013. "Effect of Cytochalasin B, Lantrunculin B, Colchicine, Cycloheximid, Dimethyl Sulfoxide and Ion Channel Inhibitors on Biospeckle Activity in Apple Tissue." Food Biophys. 8 (4): 290-6.

[16] Kurenda, A., Zdunek, A., Schlüter, O., and Herppich, W. B. 2014. "VIS/NIR Spectroscopy, Chlorophyll Fluorescence, Biospeckle and Backscattering to Evaluate Changes in Apples Subjected to Hydrostatic Pressures." Postharvest Biol. Technol. 96: 88-98.

[17] Bewley, J. D., and Black, M. 1982. Physiology and Biochemistry of Seeds in Relation to Germination: Viability, Dormancy and Environmental Control. Heidelberg, Berlin: Springer.

[18] Rodrigues, S., Braga, R. A., Enes, A. M., Batista, C. A., and Dal Fabbro, I. M. 2005. "Effect of Moisture on the Determination of the Biological Activity of Bean Seeds (Phaseolus vulgaris L.) Using Dynamic Speckle Images." Brazilian Journal of Storage 30: 135-9. (in Portuguese)
[19] Schneider, C. A., Rasband, W. S., and Eliceiri, K. W. 2012. "NIH Image to ImageJ: 25 Years of Image Analysis." Nat. Methods 9 (7): 671-5.

[20] Ferreira, D. F. 2011. "Sisvar: A Computer Statistical Analysis System." Ciência e Agrotecnologia 35 (6): 1039-42.

[21] R Development Core Team. 2013. R: A Language and Environment for Statistical Computing. Viena, Austria: R Foundation for Statistical Computing.

[22] Brazil Ministry of Agriculture, Livestock and Supply. 2009. "Rules for Seed Analysis." Ministry of Agriculture, Livestock and Supply, MAPA/ACS, Brazil. Accessed September 2016. http://www.agricultura.gov.br /arq_editor/file/2946_regras_analise_sementes.pdf. (in Portuguese)

[23] Rodrigues, S., Braga, R. A., Rabelo, G. F., Dal Fabbro, I. M., and Enes, A. M. 2007. "Application of the Dynamic Speckle in the Analysis of Bean Seeds (Phaseolus vulgaris L.)." Brazilian Journal of Agroindustrial Products 9: 119-24. (in Portuguese) 